\title{
Exploiting Electron Beam Interactions with Ultralow Energy Excitations for Nanoscale Analysis of Complex Optical and Biological Systems
}

Jordan Hachtel $^{1}$, Andrea Konecna ${ }^{2}$ and F. Javier Garcia de Abajo ${ }^{2}$

${ }^{1}$ Oak Ridge National Laboratory, Oak Ridge, Tennessee, United States, ${ }^{2}$ ICFO - The Institute of Photonic Sciences, Castelldefels, Catalonia, Spain

The newest generation of monochromated scanning transmission electron microscopes (STEM) have led to an order-of-magnitude increase in energy resolution for electron energy loss spectroscopy (EELS) [1], which has opened the door for direct analysis of mid-infrared phenomena. Beyond simply accessing phonons and molecular vibrations, these low energy excitations interact with the spatial-resolution and various flavors of momentum-resolution in the STEM to allow for new types nanoscale experiments that were not effective or not possible for higher energy phenomena. In this talk, I will focus on two such types of experiment: aloof beam analysis of fragile biological samples, and momentum-resolved analysis of phonons and phonon-polaritons in crystalline solids.

'Aloof' spectroscopy refers to placing the beam on a trajectory where it passes close to the sample without directly intersecting with it. The evanescent field of the electron beam can still interact with the sample over large distances, and critically, this interaction strength is inversely proportional to the energy of the excitation (as shown in Figure 1) [2]. This has deep implications for biological specimens, in which UV excitations begin to break apart or alter the material, as it allows us to define a regime where the infrared vibrational excitations can be efficiently detected, without inducing significant beam damage [3]. As a result, we can perform low-damage spatially resolved analysis of biological specimens and perform the direct compositional analysis of intracellular structure in whole-cell biological samples.

Momentum-resolved STEM-EELS is a well-known technique in electron microscopy: most commonly this is performed by directly defining the Brillouin zone with the diffracted Bragg disks at low convergence angles and using the EELS entrance aperture to select individual q-values [4], but one can also examine the coherent interference between propagating phenomena and their reflections off of edges to identify specific wave-vector values in quasiparticle dispersions [5]. The addition of ultrahigh energyresolution allows us to bring momentum-resolved EELS to the nanoscale in a unique way by applying these techniques to infrared phonons and phonon-polaritons. Since the energy-resolution does not influence the momentum-resolution we can operate with simultaneously high spatial/spectral/momentum resolution and access phonon dispersion curves with a nanometer-scale probe. Additionally, we can access the dispersions of novel quasiparticles, such as hyperbolic phonon polaritons, and use coherent interference between the polariton and it's reflection to directly measure the dispersion of these extremely short (going down to $<100 \mathrm{~nm}$ ) wavelength quasiparticles (Figure 2) [6]. 


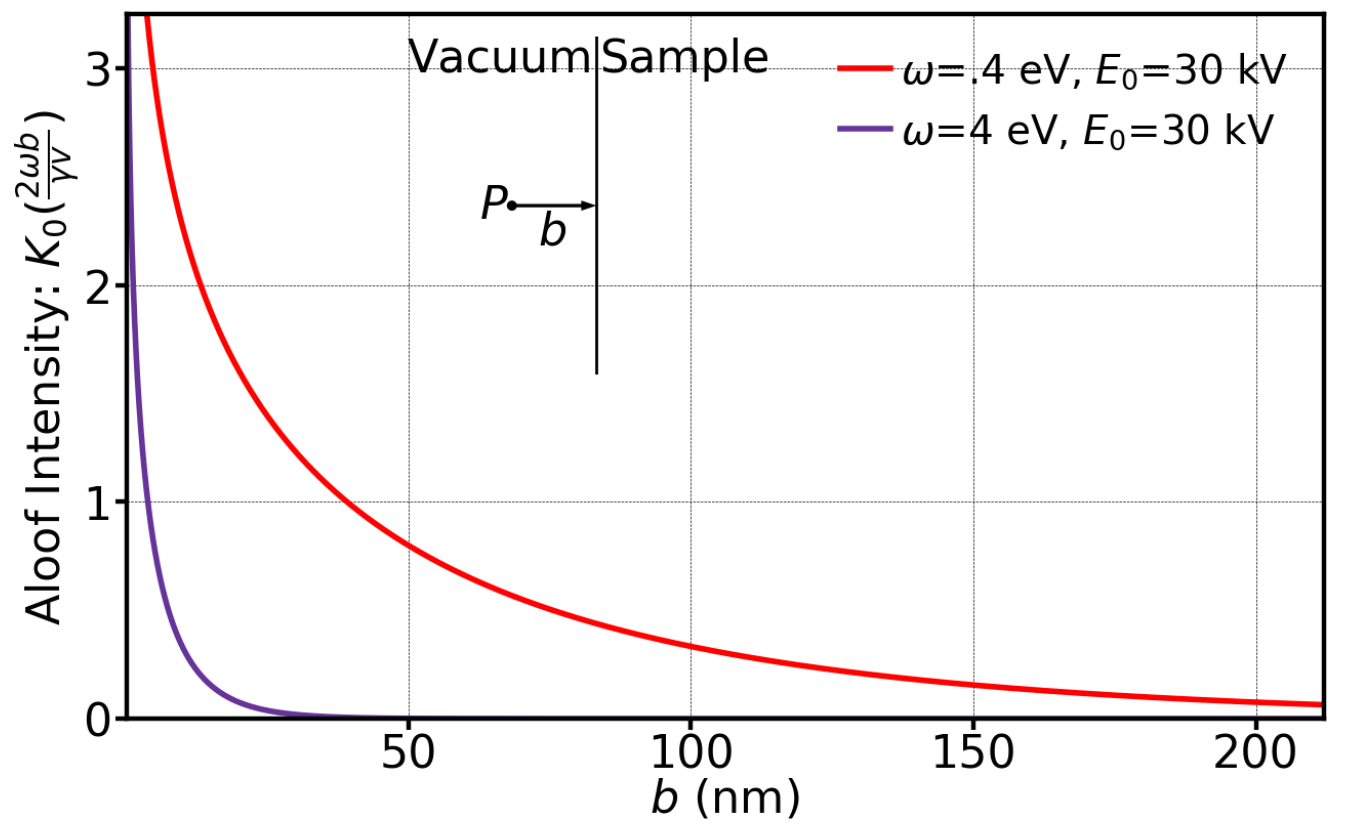

Figure 1. Intensity of the aloof interaction between the beam and the sample for an infrared energy $(0.4$ $\mathrm{eV}$ ) and an ultraviolet energy $(4 \mathrm{eV})$ as a function of distance of the aloof probe from the sample. While both interaction intensities decrease as a function of distance, below $50 \mathrm{~nm}$ but above $20 \mathrm{~nm}$ there is still significant interaction for infrared excitations, with almost no interaction for ultraviolet excitations.

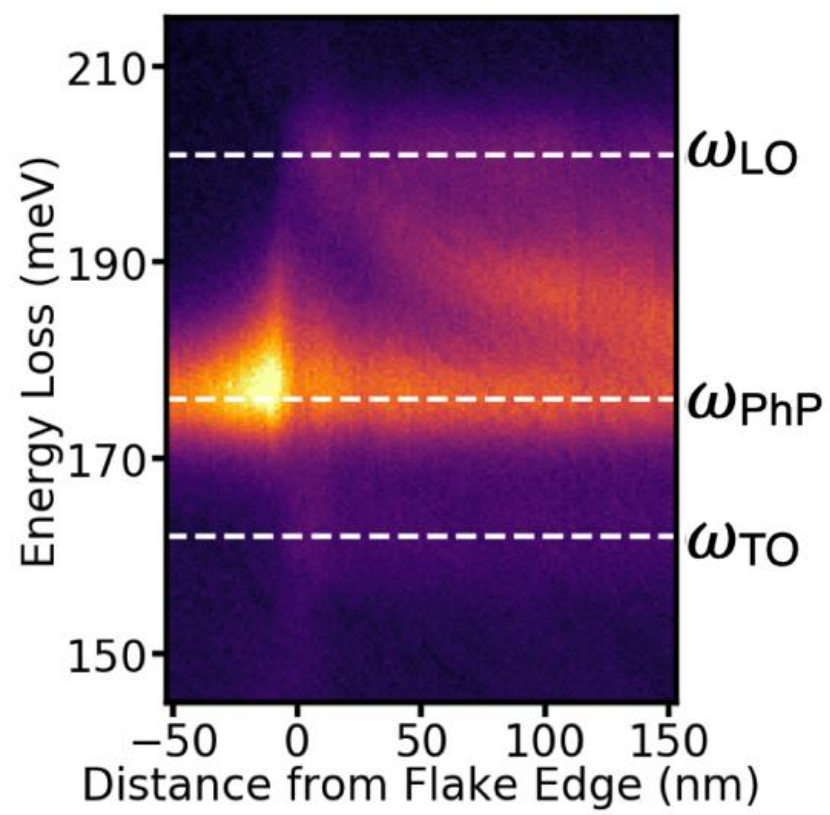

Figure 2. Line profile of the EELS intensity near the edge of a boron nitride flake. While most peaks stay at one frequency, a dispersive peak can be observed that moves between the bulk polariton frequency and the LO phonon frequency. This peak corresponds to polaritons of different wave vectors that positively interfere with their own reflections off of the edge, allowing us to directly measure the frequency and the wavevector of the entire polariton dispersion. 


\section{References}

[1] OL Krivanek et al., Ultramicroscopy 203 (2019), p. 60

[2] RF Egerton, Ultramicroscopy 159 (2015), p. 95

[3] JA Hachtel et al., Science 363 (2019), p. 525

[4] FS Hage et al., Science Advances 4 (2018), eaar77495

[5] Y Li, Science Bulletin. 65 (2020), p. 820

[6] Research conducted at the Center for Nanophase Materials Sciences, which is a DOE Office of Science User Facility. Work was performed, in part, using instrumentation within ORNL's Materials Characterization Core provided by UT-Battelle, LLC, under Contract No. DE-AC05- 00OR22725 (J.C.I.) with the DOE, and sponsored by the Laboratory Directed Research and Development Program of Oak Ridge National Laboratory, managed by UT-Battelle, LLC, for the U.S. Department of Energy. 